\title{
Automated Recovery of Issue-Commit Links Leveraging Both Textual and Non-textual Data
}

\author{
Pooya Rostami Mazrae \\ Computer Engineering Department \\ Sharif University of Technology \\ prostami@ce.sharif.edu
}

\author{
Maliheh Izadi \\ Computer Engineering Department \\ Sharif University of Technology \\ maliheh.izadi@sharif.edu
}

\author{
Abbas Heydarnoori \\ Computer Engineering Department \\ Sharif University of Technology \\ heydarnoori@sharif.edu
}

\begin{abstract}
An issue report documents the discussions around required changes in issue-tracking systems, while a commit contains the change itself in the version control systems. Recovering links between issues and commits can facilitate many software evolution tasks such as bug localization, defect prediction, software quality measurement, and software documentation. A previous study on over half a million issues from GitHub reports only about $\mathbf{4 2 . 2 \%}$ of issues are manually linked by developers to their pertinent commits. Automating the linking of commit-issue pairs can contribute to the improvement of the said tasks. By far, current state-of-the-art approaches for automated commit-issue linking suffer from low precision, leading to unreliable results, sometimes to the point that imposes human supervision on the predicted links. The low performance gets even more severe when there is a lack of textual information in either commits or issues. Current approaches are also proven computationally expensive.

We propose Hybrid-Linker, an enhanced approach that overcomes such limitations by exploiting two information channels; (1) a non-textual-based component that operates on non-textual, automatically recorded information of the commit-issue pairs to predict a link, and (2) a textual-based one which does the same using textual information of the commit-issue pairs. Then, combining the results from the two classifiers, Hybrid-Linker makes the final prediction. Thus, every time one component falls short in predicting a link, the other component fills the gap and improves the results. We evaluate Hybrid-Linker against competing approaches, namely FRLink and DeepLink on a dataset of 12 projects. Hybrid-Linker achieves $90.1 \%, 87.8 \%$, and $88.9 \%$ based on recall, precision, and F-measure, respectively. It also outperforms FRLink and DeepLink by $31.3 \%$, and $41.3 \%$, regarding the F-measure. Moreover, the proposed approach exhibits extensive improvements in terms of performance as well. Finally, our source code is publicly available.
\end{abstract}

Index Terms-Link Recovery, Issue Report, Commit, Software Maintenance, Machine Learning, Ensemble Methods

\section{INTRODUCTION}

Issues and commits are two software artifacts commonly used for various tasks in software hosting platforms such as GitHub, Jira, and Bugzilla. Issue reports encapsulate user discussions around different aspects of a software, as a sort of documentation. Commits contain source code changes required to fix bugs, add features, improvements, etc discussed in the issues. Issues are usually reported in bug-tracking systems such as Bugzilla or Jira, on the other hand, corresponding commits are stored in version control systems such as GitHub [1]. There are also cases that they are both maintained in one system. When a developer commits a change in a project, it is a good practice to mention the issue in the commit to document the relationship between the two. However, it is seldom the case due to the deadline's pressure, lack of motivation, etc. [1]. To quantify the prevalence of missing issue-commit links, Ruan et al. [2] analyzed over half a million issues from GitHub. They report only $42.2 \%$ of issues were linked to corresponding commits. Recovering issue-commit links is deemed important for improving bug prediction solutions [2], [3], bug assignment [4], feature location techniques [5], and other software maintenance tasks. It can also be used to evaluate software maintenance efforts and quality [6]. Thus, an automated method for recovering links between issues and their corresponding commits can be of high value.

The first challenge for such an approach is to use a proper dataset of True and False Links between issues and commits. True Links are the correct links between issues and their related commits. All the other combinations of links can be considered False Links. Current approaches build these links manually. This affects the reliability of results. Moreover, some issues have more than one related commit. An automatic solution to recovering True Links should be able to handle these relationships. Another important aspect is the performance of proposed approaches. Current studies mostly focus on the precision and recall scores of the predictions. However, the prediction time and complexity of the models are also important.

In this work, we introduce a novel approach, named HybridLinker to address the above-mentioned problems. HybridLinker exploits both textual and non-textual data to achieve higher performance. Textual information includes the issue title, description, code difference, and commit messages. Nontextual information consists of various characteristics of an issue and commits, such as the author of an issue, the committer, commit time, type of an issue (bug, feature, task), and state of a issue (open, closed, or resolved). We first identify all the relevant information and then perform feature engineering to extract the most important ones. The reason for incorporating non-textual data is to enable Hybrid-Linker to exploit this knowledge when there is little textual information available (e.g., there is no commit messages), or there are few similarities between the description of an issue and textual information of a commit. We train a hybrid model consisting of two classifiers and a module to achieve the best linear 
composition of these classifiers. The non-textual component is an ensemble of two classifiers. The textual component is created using TF-IDF word embeddings and a single classifier. We evaluated Hybrid-Linker against two baseline methods, FRLink and DeepLink for 12 projects with different characteristics. In summary, our contributions are as follows:

- Proposing an automatic approach, called Hybrid-Linker, for recovering the links between issues and commits using a hybrid model of classical classifiers.

- Our results show that Hybrid-Linker outperforms the competing approaches, FRLink and DeepLink, by 31.3\%, and $41.3 \%$ respectively, regarding the F-measure. Moreover, our proposed approach shows extensive improvements in terms of required training time.

- Finally, we release our source code and data publicly ${ }^{1}$

\section{Motivating ExAmple}

Here, we illustrate an example as the motivation for enhancing automatic link recovering approaches between issues and commits. Figure $1 \mathrm{a}$ is an example of an issue ${ }^{2}$ Figure $1 \mathrm{~b}$ shows an example of a commi ${ }^{3}$ related to the abovementioned issue. The issue and the commit are selected from Flink project. Apache Flink is an open-source, unified streamprocessing and batch-processing framework developed by the Apache Software Foundation. An issue has different fields like type, status, release note, description, created date, updated date, and resolved data. A commit contains commit message, committer ID, author ID, name of changed files, and Diff of changed files. Note that other information such as comments and code snippets attached to some issues do not always exist.

As shown, there is no compelling similarity between the text of issue description, its release note and the respective commit message. Due to lack of similarity in textual information of this issue and commit, FRLink approach fails to discover the True Link between them [6], Moreover, DeepLink [2] approach also struggles to identify this link as there is no code snippet in the description section of the issue. Thus, DeepLink will find little semantic relation between the issue and the source code in this commit. To address these problems, we propose to extract knowledge from both textual and non-textual channels of issues and commits. Then combine this information in a hybrid model to train stronger link recovery models.

\section{PROPOSED APPROACH}

In this section, we present the main steps of our approach, namely: (1) data crawling, (2) data preparation, (3) feature engineering, (4) model training, and (5) linear accumulator hyper-tuning. Figure 2 illustrates an overview of the approach and the following provides a detailed description of each of the five aforementioned steps.

1 https://github.com/MalihehIzadi/hybrid-linker

2 https://issues.apache.org/jira/browse/FLINK-17012

3 https://bit.ly/2PCsQu6
Alink / FLINK-17012

Expose stage of task initialization

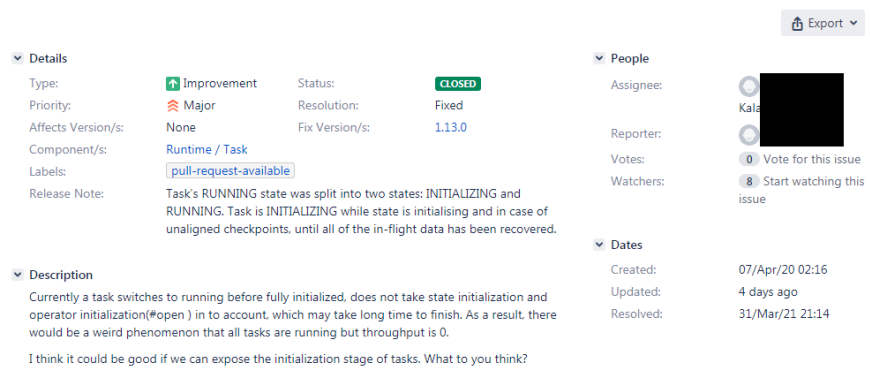

(a) Example of an issue

[FLINK-17012][webui] Added support of RECOVERING state

\& master $(\# 15462) \bigcirc$ release-1.13.0-rc1

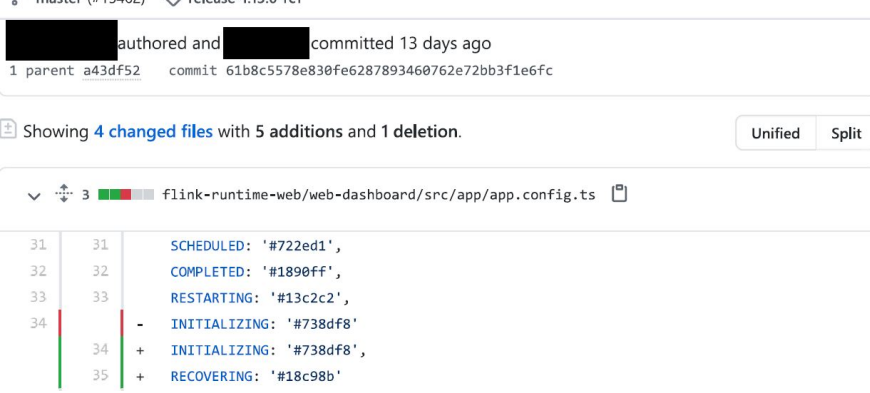

(b) Example of part of a commit

Fig. 1: Example of a True Link between an issue and a commit

\section{A. Data Crawling}

While we utilize a dataset from Claes et al. [7], this dataset does not satisfy our needs for the approach. More specifically, we aim to incorporate the code diff data of the commits into the solution, which is missing from the shared dataset due to the large volume of such data. To be able to crawl the excessively large code diff data, we first reduce the number of projects to a sample of 12 projects. Then, for the sampled projects, we crawl the missing data from the projects' code bases to complement the dataset. Moreover, the dataset is provided in a segmented state (separated commits and issues). To uniformize it, for each project, we concatenated segments of data.

\section{B. Data Preparation}

In this step, we prepare the dataset from two aspects. In the link generation process, we generate the data points, i.e., the issue-commit pairs. These data points are assigned with True Link labels when the link between the issue and its paired commit is in place and False Link otherwise, i.e., the issue and commit are irrelevant. We also perform textual data preprocessing techniques on the textual data of issues and commits separately to prepare them for feature engineering. The following elaborates on these two data preparation steps.

1) Link Generation: In the dataset, there are instances of issues and commits that are already linked by the developers, which means they are established and validated as True Links. We take such pairs of issue-commits as data points with the 


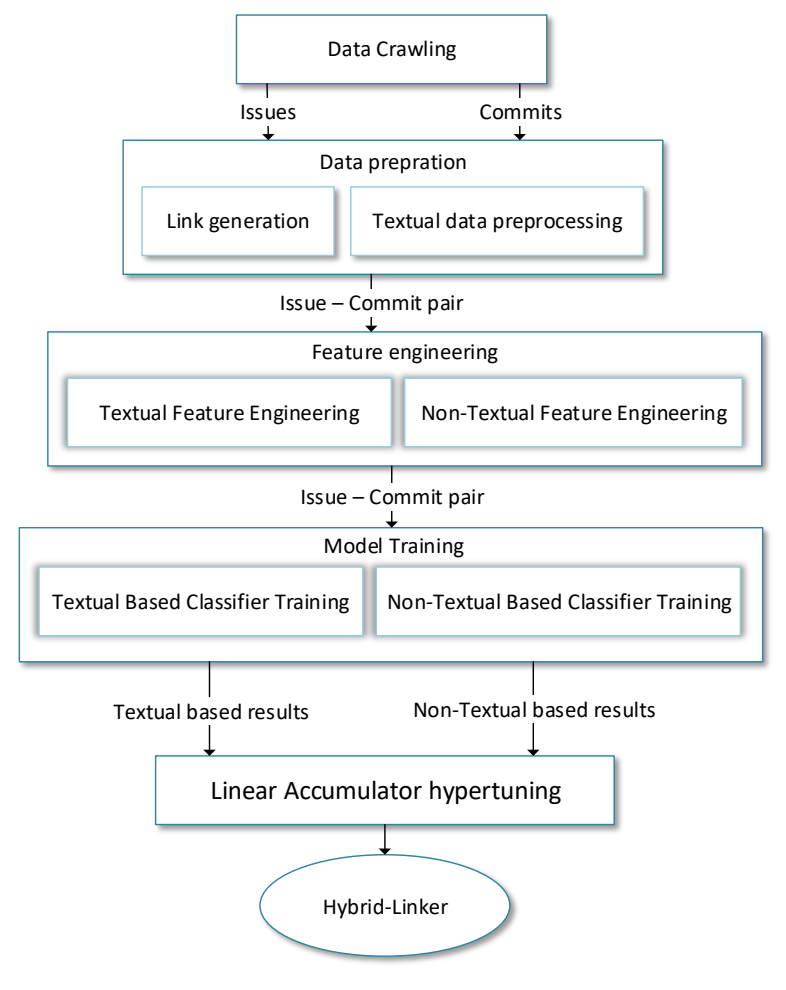

Fig. 2: Overview of the proposed approach

True Link label. To train the classifiers, we need to provide the model with data points labeled as False Link as well. However, such data points are not explicitly included in the dataset. Thus, we need to generate them such that their label, False Link, is ensured. To do so, we pair the commits that are already linked to an issue by the developers with any issue other than the ones they are already linked to. Since the commits only make a True Link with the issues selected by the developers, pairing them with any other issue makes a False Link.

However, taking all the generated links as False Link data points makes the dataset extremely imbalanced. To perceive the severity of this problem in the context of a project, consider a project with $c$ number of commits that are already linked to an issue by the developers. If the same project contains $i$ number of issues, $c$ of which are already linked to the aforementioned commits. For each commit already linked to an issue, there are $i-1$ issues each posing as a potential False Link pair for the commit. Hence, the number of False Link data points adds up to $c *(i-1)$ issue-commit pairs.

To address this issue, we use the criteria used by previous work [2], [6] to generate the False Links. Thus we compare the two relevant submission dates of a commit with the three date attributes in an issue report and construct a new False link if the commit is submitted seven days before or after any of these three issue attributes. Table I presents the information of the resultant dataset. As shown in this table, even after employing the above-mentioned criteria, the number of False Links is much larger than those of True Links for all projects.
TABLE I: Selected projects' information

\begin{tabular}{c|cc|c|ll}
\hline Project & \#Issues & \#Commits & \#Stars & $\begin{array}{l}\text { \#True } \\
\text { Links }\end{array}$ & $\begin{array}{l}\text { \#False } \\
\text { Links }\end{array}$ \\
\hline Beam & 9133 & 28824 & 4300 & 5750 & 1505559 \\
Flink & 15655 & 26517 & 14500 & 14472 & 4850083 \\
Freemarker & 127 & 4235 & 604 & 177 & 382 \\
Airflow & 6511 & 10196 & 18800 & 5295 & 1030733 \\
Arrow & 7509 & 6179 & 6400 & 5252 & 1006904 \\
Netbeans & 3705 & 19184 & 1500 & 1369 & 129639 \\
Ignite & 12495 & 32930 & 3500 & 9997 & 2087327 \\
Isis & 2264 & 15284 & 580 & 8486 & 260259 \\
Groovy & 9117 & 30478 & 3900 & 8851 & 457876 \\
Cassandra & 15413 & 31491 & 6300 & 146 & 40415 \\
Ambari & 25162 & 38872 & 1400 & 35589 & 8891881 \\
Calcite & 3740 & 6934 & 2100 & 3058 & 201106 \\
\hline
\end{tabular}

TABLE II: Code term patterns introduced in FRLink [6]

\begin{tabular}{ccc}
\hline Type & Example & Regular Expression \\
\hline C_notation & OPT_INFO & {$[\mathrm{A}-\mathrm{Za}-\mathrm{z}]+[0-9]^{*}{ }^{*}$} \\
Qualified name & op.addOption & {$[\mathrm{A}-\mathrm{Za}-\mathrm{z}]+[0-9]^{*}[\backslash .]_{+}^{+}$} \\
CamelCase & addToList & {$[\mathrm{A}-\mathrm{Za}-\mathrm{z}]+.^{*}[\mathrm{~A}-\mathrm{Z}]+.^{*}$} \\
UpperCase & XOR & {$[\mathrm{A}-\mathrm{Z} 0-9]+$} \\
System variable & $-\mathrm{cmd}$ & $-+[\mathrm{A}-\mathrm{Za}-\mathrm{z} 0-9]+.+$ \\
Refrence expression & std::env & {$[\mathrm{a}-\mathrm{ZA}-\mathrm{Z}]+[:]\{2\} .+$,} \\
\hline
\end{tabular}

To further alleviate the imbalanced nature of this dataset, we apply a common data balancing technique. More specifically, we randomly select the same number of False Links as the True Links in each project, to provide our classifier with completely balanced datasets.

2) Textual Data Preprocessing: The resultant dataset contains textual and non-textual data on issues and commits from the sampled projects. The textual data contains both natural language text such as issue title, issue description and commit message, and the code diff.

We first clean and preprocess the input textual data. We perform the three commonly-used strategies of tokenizing, removing stop words, and stemming on the natural language text data as the preprocessing step. These preprocessing actions not only reduce the vocabulary size, which in turn makes the feature set a compact one, but also they integrate different forms of words by replacing them with their roots.

As for the diff data, while they do include multiple lines of code per sample, only the identifiers, i.e., method and variable names, carry valuable information about the changes in a commit. That is because many of the keywords and commonly used method calls in the diff appear all over the code without indicating the purpose of the code snippet, while identifiers, if named according to the software development guidelines, refer to their purpose, role, and/or task. Hence, we aim to extract only the identifiers through the use of code term patterns. The code term patterns we employ are the ones previously used by Sun et al. [6] and Ruan et al. [2] (defined in Table II).

\section{Feature Engineering}

We leverage both textual and non-textual data to improve the results of the True Link prediction task. However, the features in the textual and non-textual feature vectors are not 
equally valuable in terms of being determinative of a True Link. In the textual data context, there might be distinct words throughout the dataset that appear in a significant number of the data points. This signifies that they are simply common tokens throughout the project and can not be considered as the indicator of the subject of a commit. In the non-textual context, this problem manifests itself in highly correlated columns of data or even almost identical ones. There is also the case of almost empty columns in which the data is null-valued more often than not.

This makes the feature vector unnecessarily extensive, which makes it harder for the classifier models to converge due to the multitude of parameters they are to optimize. Even if the classifier does converge and yield better results with such data included, the improvement is negligible and unjustifiable when evaluated against the computational costs. For these reasons, we perform a feature engineering process on both the textual and non-textual data to reduce the size of feature vectors and keep both the performance of the solution and the computational costs of the model optimal. The feature engineering processes performed are detailed in the following.

1) Textual Feature Engineering: We employ the widely used data modeling technique, $T F-I D F$, which captures the importance of the tokens based on probabilistic measures over the dataset. This data modeling technique computes the term frequency of each term (token) in each document and document frequency of each term over the dataset and combines the former with the inverse of the latter to calculate a measure of importance for each term in the dataset. The higher the value of the TF-IDF measure for a term, the more probable the term is to contribute to the label prediction.

We apply the TF-IDF technique on natural language textual data of commits and issues and the code diff textual data separately. This generates three vectors of TF-IDF features for each data point. Then, we concatenate the resultant vectors and construct one textual feature vector per data point. The reason for such an approach is that the information and vocabulary in issues and commits inherently differ. Moreover, according to our experiments, this approach leads to higher accuracy compared to when we combine the three input data first and then apply the TF-IDF technique on the concatenated text. Our textual features are a commit's Message and Diff, and an issue's Summary and Description. Note that we have also applied Word2 Vec and Doc2Vec techniques, however, TF-IDF embeddings produced the best result.

2) Non-textual Feature Engineering: In the case of highly correlated columns, reducing them to a single column can improve the computational costs of the model training process by limiting the number of optimization parameters, while preserving the performance of the classifier. We extensively inspect the dataset for such strongly correlated columns among commit features an issue features by calculating the similarity and correlation among the columns of similar types. We discover that among the issue data columns, over $99 \%$ of the data points have the same value as the reporter and the creator. This makes these two columns practically duplicate. Hence, we drop one and keep the other. We also detect that over $65 \%$ of the data points have the same author and committer in their commit data columns. As we believe a similarity of $65 \%$ is not high enough to justify the omission of one of the columns, so we keep both columns in the dataset.

Since the categorical data will be converted to a one-hot model, each distinct value in the categorical data column will serve as a Boolean feature. Thus, the multitude of distinct values in a categorical column results in an over-complicated feature vector with too many features but very few true points, also known as a sparse matrix. To avoid such an occurrence, we study the histograms of the categorical data and discovered that due to differences in labeling style across projects, the distinct values of the commit_status and issue_type columns can be mapped to two reduced sets of values. For commits, status values was reduced from a set of 11 statuses to three main categories of open, closed, and resolved. We also reduced the set of 15 distinct values of issue types to three main categories of task, new feature, and bug.

While there are two columns of highly correlated dates for issues, namely the create_date and the update_date, these dates prove as important features for the prediction of True Links. The same goes for the author_time_date and the commit_time_date among the data of the commits. We keep these columns intact to the dataset.

Finally, we drop the columns which have a significant number of null values. After one-hot transformation of the categorical data, we calculate the correlations among all the columns, including the label column, for issues and commits separately. This is to verify that there are no correlations among the features and target column. After it is verified that the dataset is not biased, the resultant commit and issue feature vectors are concatenated to compose a single feature vector for each data point. Our non-textual features are commit time, authoring time, author hash, and commit hash of commits. We also include updated date, created date, status (closed, open, resolved), issue type (bug, new feature, task) and creator hash from issue reports.

\section{Model Training}

We aim to keep the classifier model simple to lower the computational costs of training and prediction. We believe one can improve the prediction accuracy of these models by augmenting the input data. To do so, we leverage both textual and non-textual data on the commits and issues and construct a hybrid model by training two classifiers, one that operates on textual data and calculates the probability of labels, and another one that does the same using non-textual data.

1) Textual Classifier Model: As the textual classifier component, we train multiple classification models, namely a Decision Tree (DT), a Gradient Boosting (GB), a Logistic Regression (LR), and a Stochastic Gradient Descent (SGD) model to choose the model with the best performance among them. We feed these models the resultant feature vectors from Section III-C1 and train them. The trained models take as input 


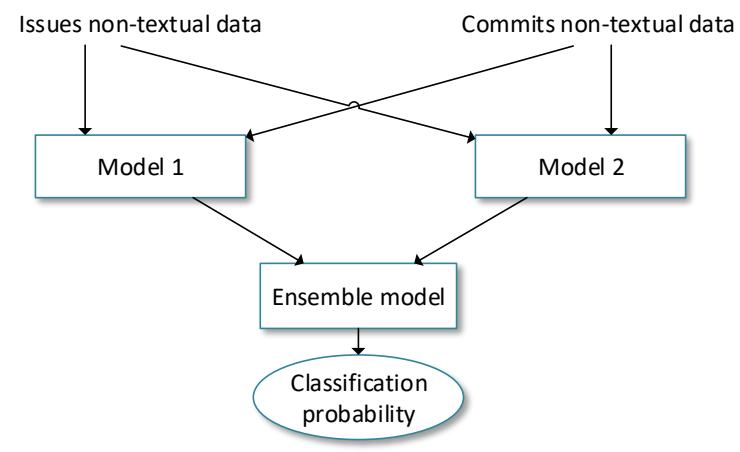

Fig. 3: Overview of ensemble models for non-textual classifier component

the processed vector of textual data and predict a label, either True or False Link for an issue-commit pair.

2) Non-textual Classifier Model: Here, we also use single and ensemble models to achieve the best results [8]. As simple classifier models, we train a Gradient Boosting [9], a Naive Bayes (NB) [10], a Generalized Linear (GL), a Random Forest (RF) [11], and a XGBoost [12] model. To construct the ensemble models, following the overview illustrated in Figure 3, we combine the models and make four ensemble models accordingly. The ensemble models are a $R F+G B$, a $G B+X G B o o s t$, a $R F+X G B o o s t$, and a $R F+G B+X G B$ oost combinations.

\section{E. Linear Accumulator Hyper-tuning}

The last step in our approach is to combine the predictions of the two classifier components and generate the final prediction on the label of the data points. To do so, we combine the predicted probability of a data point being a True Link from the two components and with a linear accumulator function, defined as the following:

$$
P_{f}=\alpha \times P_{n t}+(1-\alpha) \times P_{t}
$$

in which $P_{f}$ is the final calculated probability of a commitissue pair being a true-link, $P_{n t}$ is the probability of the same pair being a true-link according to the non-textual classifier component, and $P_{t}$ is the same probability according to the textual classifier component.

In Equation 1, $\alpha$ is the hyper-parameter by which we tune the model to produce the best results tailored to the characteristics of each project. To do so, we vary the value of $\alpha$ from 0.00 to 1.00 in 0.05 steps. The value $\alpha$ by which best results regarding the F1-score is yielded is taken as the optimal $\alpha$ value.

\section{EXPERIMENTAL DESIGN}

\section{A. Research Questions}

We define three Research Questions (RQ) to measure the effectiveness of our proposed approach. We review these questions in the following.
- RQ1: Compared to the state-of-the-art approaches, how effective is our approach in recovering the missing links between issues and commits? To answer this question, we evaluate our method's performance using the 20MAD dataset (reviewed in the next section) [7]. We use 12 projects from this dataset for training and testing our model. There are different approaches for commit-issue recovery. We use two of the state-of-the-art models, namely FRLink [6] and DeepLink [2], to compare with the proposed approach.

- RQ2: How to combine the two components of the model to achieve the best outcome? There are different ways to combine our two models (textual and non-textual). With this question, we aim to identify the best method to build a hybrid model.

- RQ3: What is the effect of each component of the model on the outcome? As our model is constructed of different components, we assess the benefits of adding each through an ablation study. That is, we evaluate the model using each of the two components separately and then compare the results by those of the Hybrid-Linker.

\section{B. Data Selection}

As the data of previous work were not publicly shared, we utilized the dataset presented by Claes and Mantyla [7] in the MSR conference, 2020. From the Apache projects, we chose 12 based on two criteria; (1) having a repository with more than 500 stars (to have good input data for training the models), and (2) having a diverse number of issues for different projects (to be fair). As of September 2020, the number of stars for the selected projects was in the range of 580 to 18800 . The second criterion let us choose projects with different number of issues from small to large software projects. The number of issues among our projects range from a couple hundred to more than $25 \mathrm{~K}$ issues. To prepare this data for feeding our Machine-Learning-based models, we complement and transform the selected 12 projects from the 20MAD dataset as explained in Sections III-A and III-B

\section{Evaluation Metrics}

As previously used in the related work, we use three metrics of Precision, Recall, and F-measure to evaluate the performance of the approach [2], [6]. These metrics are calculated using the following equations.

$$
\begin{gathered}
\text { Precision }=\frac{T P}{T P+F P} \\
\text { Recall }=\frac{T P}{T P+F N} \\
F 1=\frac{2 \times \text { Precision } \times \text { Recall }}{\text { Precision }+ \text { Recall }}
\end{gathered}
$$

\section{Experiment Setting}

For preprocessing, we use Pandas [13] library. For training the classifiers in the non-textual component of our approach, we use $\mathrm{H} 2 \mathrm{O} . \mathrm{ai}$ [14] library. $\mathrm{H} 2 \mathrm{O}$ benefits from distributed, inmemory processing which results in faster models. It is also 
able to manage hash data better than the Sci-Kit Learn [15] library. This library can be used in different programming languages such as Python and R. We use the Python version and added a Java Runtime Environment for the backend. For the textual component, we use the Sci-Kit Learn library. It has great I/O which lets us use different types of data like Parquet and Pickle files simultaneously.

We use five-fold cross-validation to evaluate the models more thoroughly. That is, we break the data into five parts randomly, choose one as the test set and use the others for training. After repeating this process five times, iterating over the parts as the test set, we report the average as the result of evaluations. This also helps with the generalizability of the approach and avoiding the overfitting problem.

To find the best parameters for the ensemble model in the non-textual component, we perform a Random Grid search for each project of the dataset. The result of the search indicate that (n_trees $=60$, max_depth $=15$, min_rows $=2$, learn_rate $=0.1$, learn_rate_annealing $=1$ ) for the Gradient Boosting model and (n_trees $=60$, max_depth $=15$, min_rows $=2$, learn_rate $=0.1$ ) for the XGBoost model are the best choices.

To find the best parameters for the Gradient Boosting in the textual component, we perform another Random Grid search for each project of the dataset. The result of the search indicate that (n_estimators $=300$, max_features $=$ None, max_depth $=50$ and learning_rate $=0.1$ ) are the best choices.

To build the TF-IDF embedding vectors, we experiment with unigram, bigram, and union of unigram, bigram, and trigram word embedding. The best case is the union of unigram, bigram, and trigram as it finds all the important individuals and combinations of words. For TF-IDF embeddings, we set a maximum number of features to $10 \mathrm{~K}$.

For all of our experiments, we used the same machine with $32 \mathrm{~GB}$ memory and a 4-core Intel i7-7700k 4.2G processor.

The baselines here are FRLink [6] and DeepLink [2] as they achieve the state-of-the-art results in the problem of automatically recovering links between issues and their corresponding commits. FRLink uses a set of features and complementary documents such as non-source documents to learn from relevant data for recovering links [6]. They analyze and filter out irrelevant source code files to reduce data noise. On the other hand, DeepLink uses a semantically-enhanced link recovery method based on deep neural networks [2]. The authors apply a recurrent neural network on the textual information of issues and commits for training their model. They also disregard issue comments due to their length and noise. While DeepLink outperforms FRLink in terms of Fmeasure, it achieves lower recall scores [2]. Thus, we use both these techniques here as the baselines to compare our approach with. We use the replication packages provided by Ruan et al. [2] for these two model $4^{4}$ We slightly modified their input reader function to be able to read our data. Moreover, we set all the parameters as specified in the original papers.

\footnotetext{
${ }^{4}$ https://github.com/ruanhang1993/DeepLink
}

TABLE III: The average performance of models on textual data.

\begin{tabular}{l|ccc}
\hline Project & Recall & Precision & F-measure \\
\hline Decision Tree & $73.30 \%$ & $73.58 \%$ & $73.25 \%$ \\
Gradient Boosting & $\mathbf{7 8 . 2 7 \%}$ & $\mathbf{7 8 . 3 8 \%}$ & $\mathbf{7 8 . 2 5 \%}$ \\
Logistic Regression & $64.60 \%$ & $65.08 \%$ & $64.55 \%$ \\
Stochastic Gradient Descent & $63.67 \%$ & $63.91 \%$ & $63.61 \%$ \\
\hline
\end{tabular}

TABLE IV: The average performance of models on nontextual data.

\begin{tabular}{l|c|ccc}
\hline Method & Algorithm & Recall & Precision & F-measure \\
\hline \multirow{3}{*}{ Simple } & Gradient Boosting(GB) & $\mathbf{1 0 0 \%}$ & $\mathbf{9 6 . 3 4 \%}$ & $\mathbf{8 5 . 5 0 \%}$ \\
Method & Naïve Bias & $100 \%$ & $66.97 \%$ & $73.77 \%$ \\
& Generalized Linear & $100 \%$ & $88.98 \%$ & $76.18 \%$ \\
& Random Forest(RF) & $\mathbf{1 0 0 \%}$ & $\mathbf{9 8 . 2 5 \%}$ & $\mathbf{8 4 . 5 5 \%}$ \\
& XGBoost & $\mathbf{1 0 0 \%}$ & $\mathbf{9 8 . 6 7 \%}$ & $\mathbf{8 6 . 1 4 \%}$ \\
\hline \multirow{3}{*}{ Ensemble } & RF + GB & $100 \%$ & $98.29 \%$ & $86.61 \%$ \\
Method & GB + XGBoost & $\mathbf{1 0 0 \%}$ & $98.73 \%$ & $\mathbf{8 7 . 8 1 \%}$ \\
& RF + XGBoost & $100 \%$ & $\mathbf{9 9 . 2 7 \%}$ & $87.10 \%$ \\
& RF + GB + XGBoost & $100 \%$ & $98.81 \%$ & $87.77 \%$ \\
\hline
\end{tabular}

\section{RESULTS}

In this section, we answer the research questions by providing the results of the experiments. We first, compare the performance of our proposed approach with the state-of-theart ones. Next, we review the results of our investigations on how to build a hybrid model. Finally, we present the results of the ablation study to show the effectiveness and impact of each component of the proposed approach.

a) RQ1: Compared to the state-of-the-art approaches, how effective is our approach in recovering the missing links between issues and commits? To answer this RQ, we built our approach with two classifier components, a textual classifier and a non-textual one that each predict the probability of a issue-commit pair being a true-link. We plugged multiple classifier models into each of the said components and chose the models with best performances as our proposed ones.

For the textual classifier component, we fed the concatenated TF-IDF vectors to four classifier models widely used for text classification purposes and study the results to determine the best performance among the models. Table III shows the outcome of the trained models. The results indicate the best algorithm for classifying issue and commits linkage based on their textual data is the Gradient Boosting model.

For training a classifier on non-textual information, we experimented with well-known classical classifiers to identify the best classifier for our case. As seen in Table IV Gradient Boosting, Random Forest, and XGBoost have higher overall metrics results. Moreover, ensemble methods have been shown to outperform simple models. Thus, we also opt for an ensemble model of the above algorithms to identify the best combination here. Based on results in Table IV, the ensemble of Gradient Boosting and XGBoost produce the best result for our non-textual data. Table IV reports the average score of precision, recall and F-measure for each model. 
The effectiveness of our proposed method is evaluated based on three metrics, namely Precision, Recall, and F-measure. Table $\mathrm{V}$ presents a summary of the average performance of our approach across projects. According to the results, our approach achieves an average of $90.14 \%$ on Recall, $87.78 \%$ on Precision, and $88.88 \%$ of F-measure. Respectively, the lowest Recall is $84.41 \%$ for Arrow and the highest is $100 \%$ for Cassandra project. On the other hand, the lowest precision is $81.81 \%$ for Cassandra and the highest is $96.04 \%$ for Ambari.

We compare our approach with two of the competing models, namely FRLink and DeepLink. On average, our approach has $34.17 \%$ higher precision and $21.21 \%$ higher F-measure scores than FRLink. Although FRLink achieves higher recall than our proposed approach, its precision score is much lower compared to our model. Hence, Hybrid-Linker ultimately outperforms FRLink based on F-measure which is the harmonic mean of recall and precision. Moreover, obtaining high recall but low precision calls for manual assessment of the predictions. That is, a developer needs to check the predicted links and remove the incorrect ones. This adversely affects the automated feature of the approach.

Hybrid-Linker outperforms DeepLink by $50.40 \%, 26.99 \%$, and $41.34 \%$ regarding the average recall, precision, and Fmeasure. Previous studies have shown deep learning-based models tend to outperform classical machine learning models. However, as shown in a study by Hellendoorn et al. [16], it is possible to achieve better results using simple and wellengineered approaches compared with vanilla deep neural networks. According to our results, we are also able to surpass DeepLink as we carefully inspect the domain of the problem, identify and incorporate more relevant information from the non-textual channel in addition to the textual information of issues and commits. Evidently, these types of information can help boost the performance of automatic link recovery models. Our results are also compatible with those reported by Ruan et al. [2] where the overall recall score of FRlink is higher than DeepLink. However, Ruan et al. [2] originally evaluated using six projects with almost identical number of true/false links, while in this study we have included 12 projects with various number of true/false links and sizes to improve diversity of our dataset. This may cause the drop in individual scores reported in this work and Ruan et al.'s [2] study (regarding comparison with FRlink).

Furthermore, our approach uses fewer computational resources and time while training the models. For instance, pertaining the Airflow project, the required time to train Hybrid-Linker is 25 minutes, while it takes about 7 hours to train DeepLink. able $\overline{\mathrm{VI}}$ provides execution times per project. DeepLink has also reported a $5 \mathrm{X}$ overhead comparing their approach to FRLink. The large overhead of DeepLink is probably due to the complex nature of deep models. Our results indicate we can train simpler models which incorporate more relevant information, thus, achieving higher accuracy and less overhead.

We also calculated the standard deviation of the F-measure for the 12 projects in the dataset. Taking all projects into account, the standard deviations of the F-measure are 3.01, 3.92, and 4.68 for Hybrid-Linker, DeepLink, and FRLink, respectively. That is, our approach is more stable than the other two, hence proving to be a more generalizable approach.

b) RQ2: How to combine the two components of the model to achieve the best outcome? To incorporate as much information as possible and consequently boost the performance, we propose a hybrid model of our two distinct classifiers. To combine the predictions of the two components, we create a linear composition of their outputs.

Figure 4 presents the results of using different values of alpha ranging from 0 to 1 for the 12 projects under study. As can be seen, each project requires a different value of alpha. Thus, selecting a constant alpha for all projects will result in weaker results.

Table VII lists the best $\alpha$ values for each project. In most cases, $\alpha$ is above 0.5 , with the average $\alpha$ being 0.66 for all the projects. This means, interestingly, in most cases the non-textual component plays a more important role in the final decision making of Hybrid-linker. This highlights the importance of incorporating these types of information while recovering links. The only exception to this finding occurs in the Ambari project with $\alpha$ of 0.45 . This implies an approximately equal contribution of the two components of our proposed approach for this project. On the other hand, for Calcite, the best results are achieved with an $\alpha$ of 0.95 . This can be a indicator that this project lacks adequate textual information useful for recovering links.

c) RQ3: What is the effect of each component of the model on the outcome? In this section, we present the results of our ablation study on assessing the effect of each component of the proposed model. Table VIII presents a summary of our model's performance based on each project. The results indicate that on average, the performance of the textual model is lower than both the non-textual and hybrid models. The textual model also marks the highest standard deviation among the models with 5.83. Interestingly, the non-textual model outperforms the hybrid model regarding precision by $4.38 \%$. On the other hand, the standard deviation of the non-textual model is 3.10 which is slightly higher than the standard deviation of the hybrid model, 3.00. As natural language is more complex, text-based approaches may require more complex techniques to perform fairly good. The higher performance of the non-textual component is probably due to (1) having more explicit data, and (2) the advantage of ensemble models. To conclude, the hybrid model has higher recall and F-measure scores. It also obtains the lowest standard deviation regarding its performance on all the projects. This means, by employing both of the textual and non-textual components, the hybrid model achieves higher results, while preserving the stability of the proposed approach.

\section{A. Discussion}

Here, we present an example where our model successfully recovers the True Link between an issue and its corresponding commit. Table [X] summarizes the information of these 
TABLE V: Performance of the models

\begin{tabular}{|c|c|c|c|c|c|c|c|c|c|}
\hline \multirow[b]{2}{*}{ Project } & \multicolumn{3}{|c|}{ Hybrid-Linker } & \multicolumn{3}{|c|}{ DeepLink } & \multicolumn{3}{|c|}{ FRLink } \\
\hline & Recall & Precision & F-measure & Recall & Precision & F-measure & Recall & Precision & F-measure \\
\hline Beam & $85.77 \%$ & $86.22 \%$ & $85.99 \%$ & $82.63 \%$ & $55.15 \%$ & $66.15 \%$ & $100 \%$ & $50.43 \%$ & $67.05 \%$ \\
\hline Flink & $91.91 \%$ & $89.69 \%$ & $90.79 \%$ & $43.98 \%$ & $63.43 \%$ & $51.94 \%$ & $88.63 \%$ & $61.80 \%$ & $72.82 \%$ \\
\hline Freemarker & $88.89 \%$ & $91.42 \%$ & $90.14 \%$ & $95.83 \%$ & $100 \%$ & $97.87 \%$ & $97.22 \%$ & $61.40 \%$ & $75.26 \%$ \\
\hline Airflow & $87.80 \%$ & $85.72 \%$ & $86.75 \%$ & $44.54 \%$ & $64.45 \%$ & $52.67 \%$ & $94.32 \%$ & $66.77 \%$ & $78.19 \%$ \\
\hline Arrow & $84.41 \%$ & $83.71 \%$ & $84.06 \%$ & $16.85 \%$ & $44.65 \%$ & $24.47 \%$ & $99.90 \%$ & $52.14 \%$ & $68.52 \%$ \\
\hline Netbeans & $88.84 \%$ & $85.66 \%$ & $87.22 \%$ & $57.39 \%$ & $73.56 \%$ & $64.48 \%$ & $92.93 \%$ & $62.65 \%$ & $74.85 \%$ \\
\hline Ignite & $90.82 \%$ & $89.59 \%$ & $90.20 \%$ & $68.58 \%$ & $70.16 \%$ & $69.36 \%$ & $100 \%$ & $50.71 \%$ & $67.29 \%$ \\
\hline Isis & $88.13 \%$ & $89.84 \%$ & $88.98 \%$ & $47.78 \%$ & $74.80 \%$ & $58.31 \%$ & $100 \%$ & $49.39 \%$ & $66.12 \%$ \\
\hline Groovy & $89.15 \%$ & $87.79 \%$ & $88.47 \%$ & $47.65 \%$ & $62.5 \%$ & $54.07 \%$ & $94.26 \%$ & $54.83 \%$ & $69.33 \%$ \\
\hline Cassandra & $100 \%$ & $81.81 \%$ & $90 \%$ & $72.72 \%$ & $84.21 \%$ & $78.04 \%$ & $100 \%$ & $45.76 \%$ & $62.79 \%$ \\
\hline Ambari & $97.13 \%$ & $96.04 \%$ & $96.58 \%$ & $87.50 \%$ & $72.11 \%$ & $79.06 \%$ & $98.57 \%$ & $62.13 \%$ & $76.22 \%$ \\
\hline Calcite & $88.85 \%$ & $85.89 \%$ & $87.34 \%$ & $55.58 \%$ & $60.74 \%$ & $58.04 \%$ & $96.55 \%$ & $61.80 \%$ & $75.36 \%$ \\
\hline $\begin{array}{l}\text { Avg. } \\
\text { Diff from Hybrid-Linker }\end{array}$ & $90.14 \%$ & $\mathbf{8 7 . 7 8 \%}$ & $88.88 \%$ & $\begin{array}{c}60.09 \% \\
(-30.05 \%)\end{array}$ & $\begin{array}{c}68.81 \% \\
(-18.97 \%)\end{array}$ & $\begin{array}{c}62.87 \% \\
(-26.01 \%)\end{array}$ & $\begin{array}{c}96.86 \% \\
(+6.72 \%)\end{array}$ & $\begin{array}{c}53.61 \% \\
(-34.17 \%)\end{array}$ & $\begin{array}{c}67.67 \% \\
(-21.21 \%)\end{array}$ \\
\hline
\end{tabular}

TABLE VI: execution time for each project on our hardware

\begin{tabular}{clcccc}
\hline Project & $\begin{array}{l}\text { Hybrid- } \\
\text { Linker }\end{array}$ & DeepLink & Project & $\begin{array}{l}\text { Hybrid- } \\
\text { Linker }\end{array}$ & DeepLink \\
\hline Beam & $35 \mathrm{~m}$ & $19 \mathrm{~h}$ & Flink & $2 \mathrm{~h}$ & $3 \mathrm{~d}$ \\
Freemarker & $11.5 \mathrm{~s}$ & $30 \mathrm{~m}$ & Airflow & $25 \mathrm{~m}$ & $7 \mathrm{~h}$ \\
Arrow & $35 \mathrm{~m}$ & $6 \mathrm{~h}$ & Netbeans & $7 \mathrm{~m}$ & $25 \mathrm{~d}$ \\
Ignite & $22 \mathrm{~m}$ & $13.5 \mathrm{~d}$ & Isis & $28 \mathrm{~m}$ & $23 \mathrm{~h}$ \\
Groovy & $54 \mathrm{~m}$ & $13 \mathrm{~h}$ & Cassandra & $33 \mathrm{~m}$ & $6 \mathrm{~h}$ \\
Ambari & $4 \mathrm{~h}$ & $7.5 \mathrm{~d}$ & Calcite & $31 \mathrm{~m}$ & $6 \mathrm{~h}$ \\
\hline
\end{tabular}

TABLE VII: Best value of Alpha per project

\begin{tabular}{cc|cc|cc}
\hline Project & Alpha & Project & Alpha & Project & Alpha \\
\hline Beam & 0.7 & Ignite & 0.65 & Flink & 0.6 \\
Isis & 0.55 & Freemarker & 0.6 & Groovy & 0.55 \\
Airflow & 0.65 & Cassandra & 0.75 & Arrow & 0.7 \\
Ambari & 0.45 & Netbeans & 0.8 & Calcite & 0.95 \\
\hline
\end{tabular}

two artifacts. Although there are few similarities in textual information, the baselines and our textual component are unable to recognize this connection. However, our non-textual component compensates for this shortcoming and predicts the correct connection. As it is shown, our model is capable of correct predictions both (1) when there is little textual information available or (2) when there is no explicit relation among the text of the two artifacts. Note that non-textual data are often available as they are automatically recorded.

\section{B. Threats to Validity}

Here we discuss the threats to the validity of our work, organized into internal, external, and construct validity.

a) Internal Validity: Internal validity is the extent to which a piece of evidence supports a claim about cause and effect, within the context of a particular study [17]. The first threat to the internal validity of our study is the True Link trustworthiness and False Link trustworthiness in our dataset. In the case of building True Links, we have used the links provided by Claes and Mantyla in [7]. Although this dataset is validated by the authors [7], incorrect links may still be present due to human error. Any combination other than a True Link can be considered a False Link. However, due to the diversity and multitude of choices for creating False
Links, we had to employ several constraints as explained in Section IV-B These constraints affect our results. According to previous studies, if an issue is related to a commit, there is a higher chance it will be answered/solved by a commit within seven days. Thus, by creating different combinations of False Links within seven days, we aim to create a more relevant and appropriate False Link dataset for training the models. Lastly, data balancing is an important issue to keep in mind. Although one can easily create a large number of False Links, lack of enough True Links adversely affects the performance of classifiers. To tackle this problem, we balanced the dataset by selecting a random subset of the over-presented class before training. Other balancing techniques are also viable.

b) External Validity: External validity is concerned with the generalizability of the approach and results [17]. In that regard, the dataset used in this study affects the outcome of the models. The size and quality of the data play an important role in having a good issue and commit link predictor. We addressed this threat by evaluating our approach against data from multiple projects and studying the results. As discussed in Section $\mathrm{V}$, the lower standard deviation achieved by HybridLinker indicates that results from this approach are more stable across projects. That is, the approach is more generalizable than the state-of-the-art baselines and produces results in an expected range when applied on data from different projects.

c) Construct Validity: Construct validity is concerned with the evaluation of the models [17]. Similar to previous work [1]-[3], [6], [18], we use precision, recall, and Fmeasure to evaluate the performance of our approach. To evaluate our proposed model more fairly, we also use fivefold cross-validation in all model evaluation steps of the study and report the average of the metrics. By breaking data into five smaller chunks and re-evaluating the model, we ensure that all of the data has been used for training and testing.

\section{RELATED WORK}

In this section, we review the related studies with the purpose of linking issues to their corresponding commits. We categorize these approaches into three major groups of 

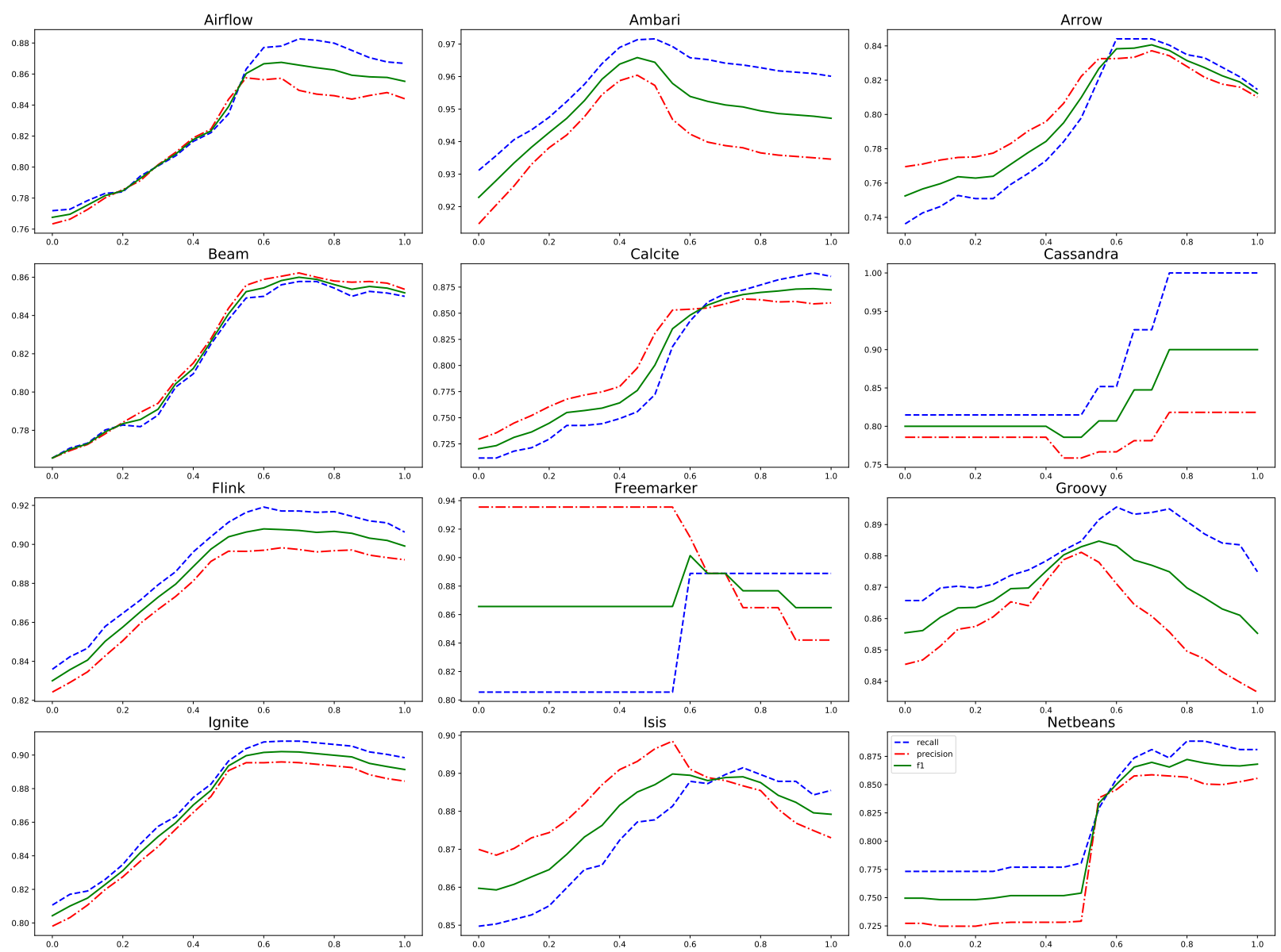

Fig. 4: Tuning Alpha per project

TABLE VIII: Results of the ablation study

\begin{tabular}{c|ccc|ccc|ccc}
\hline & \multicolumn{3}{|c|}{ Hybrid method } & \multicolumn{3}{c|}{ Textual-based } & \multicolumn{3}{c}{ Non-textual-based } \\
Project & Recall & Precision & F-measure & Recall & Precision & F-measure & Recall & Precision & F-measure \\
\hline Beam & $\mathbf{8 5 . 7 7 \%}$ & $86.22 \%$ & $\mathbf{8 5 . 9 9 \%}$ & $76.35 \%$ & $76.35 \%$ & $76.35 \%$ & $84.64 \%$ & $\mathbf{8 6 . 4 6 \%}$ & $85.54 \%$ \\
Flink & $\mathbf{9 1 . 9 1 \%}$ & $89.69 \%$ & $\mathbf{9 0 . 7 9 \%}$ & $82.52 \%$ & $82.52 \%$ & $82.51 \%$ & $89.77 \%$ & $\mathbf{9 0 . 3 2 \%}$ & $90.05 \%$ \\
Freemarker & $88.89 \%$ & $\mathbf{9 1 . 4 2 \%}$ & $\mathbf{9 0 . 1 4 \%}$ & $87.32 \%$ & $88.10 \%$ & $87.27 \%$ & $\mathbf{9 3 . 9 3 \%}$ & $86.11 \%$ & $89.85 \%$ \\
Airflow & $\mathbf{8 7 . 8 0 \%}$ & $85.72 \%$ & $\mathbf{8 6 . 7 5 \%}$ & $76.30 \%$ & $76.30 \%$ & $76.30 \%$ & $81.50 \%$ & $\mathbf{9 1 . 5 2 \%}$ & $86.22 \%$ \\
Arrow & $\mathbf{8 4 . 4 1 \%}$ & $83.71 \%$ & $\mathbf{8 4 . 0 6 \%}$ & $75.01 \%$ & $75.10 \%$ & $75.02 \%$ & $74.98 \%$ & $\mathbf{9 0 . 4 0 \%}$ & $81.97 \%$ \\
Netbeans & $\mathbf{8 8 . 8 4 \%}$ & $85.66 \%$ & $87.22 \%$ & $74.64 \%$ & $74.76 \%$ & $74.62 \%$ & $82.37 \%$ & $\mathbf{9 5 . 5 3 \%}$ & $\mathbf{8 8 . 4 6 \%}$ \\
Ignite & $\mathbf{9 0 . 8 2 \%}$ & $\mathbf{8 9 . 5 9 \%}$ & $\mathbf{9 0 . 2 0 \%}$ & $79.99 \%$ & $80 \%$ & $79.99 \%$ & $90.07 \%$ & $88.60 \%$ & $89.33 \%$ \\
Isis & $\mathbf{8 8 . 1 3 \%}$ & $89.84 \%$ & $\mathbf{8 8 . 9 8 \%}$ & $86.30 \%$ & $86.32 \%$ & $86.30 \%$ & $87.08 \%$ & $\mathbf{9 0 . 0 4 \%}$ & $88.53 \%$ \\
Groovy & $\mathbf{8 9 . 1 5 \%}$ & $87.79 \%$ & $\mathbf{8 8 . 4 7 \%}$ & $85.62 \%$ & $85.6 \%$ & $85.60 \%$ & $81.20 \%$ & $\mathbf{9 1 . 4 5 \%}$ & $86.02 \%$ \\
Cassandra & $\mathbf{1 0 0 \%}$ & $81.81 \%$ & $90 \%$ & $81.36 \%$ & $81.45 \%$ & $81.38 \%$ & $84.37 \%$ & $\mathbf{1 0 0 \%}$ & $\mathbf{9 1 . 5 2 \%}$ \\
Ambari & $\mathbf{9 7 . 1 3 \%}$ & $\mathbf{9 6 . 0 4 \%}$ & $\mathbf{9 6 . 5 8 \%}$ & $92.10 \%$ & $92.12 \%$ & $92.10 \%$ & $93.37 \%$ & $95.86 \%$ & $94.73 \%$ \\
Calcite & $\mathbf{8 8 . 8 5 \%}$ & $85.89 \%$ & $87.34 \%$ & $72.47 \%$ & $72.48 \%$ & $72.46 \%$ & $83.55 \%$ & $\mathbf{9 3 . 2 7 \%}$ & $\mathbf{8 8 . 1 4 \%}$ \\
\hline Avg. & $\mathbf{9 0 . 1 4 \%}$ & $87.78 \%$ & $\mathbf{8 8 . 8 8 \%}$ & $80.83 \%$ & $80.92 \%$ & $80.82 \%$ & $85.57 \%$ & $\mathbf{9 1 . 6 3 \%}$ & $88.36 \%$ \\
\hline
\end{tabular}

TABLE IX: An example of a True Link prediction

\section{Issue Information}

created_date: $2014-12-08$

updated date: $2014-12-10$

summary: "copy method logicalaggregate not copying indicator value properly"

description: " $\{\{$ copy $\}\}$ method $\{\{$ logicalaggregate $\}$ \} not take value $\{\{$ indicator $\}\}$ boolean input parameters object itself.'

bug: 1 , new feature: 0 , task: 0

creator_key: 59f263ad6f803c44dd2c8d5a716571218af230278

closed: 1 , open: 0 , resolved: 0
Commit Information

author_time_date: 2014-12-10

commit time date: $2014-12-10$

message: "[ calcite-511 ] copy method logicalaggregate not copying indicator value properly fixes \# 26"

DiffCode: "logical aggregate .java logical aggregate .java logical aggregate .java trait set .contains applicable convention none logical aggregate rel input immutable bit set group set immutable bit set group set aggregate call agg call logical aggregate get cluster group set logical aggregate get cluster group set group set agg call"

author: a0465f128099ac027df4ee3910ee43aa66ad154b committer: 0dc204239e 76b8945e61c77525ac8f7386763a23 
heuristic-based, Machine-Learning-based, and Deep-learningbased studies.

a) Heuristic-based approaches: These studies simply define a set of heuristics to find the links between issues and commits. ReLink [19], MLink [20], and PaLiMod [18] fall into this category. Wu et al. [19], introduced ReLink, an approach that builds on top of traditional heuristics for creating True Links. The traditional heuristics used in this work mostly rely on hints or links developers leave about bug fixes in changelogs. For instance, they search for keywords such as 'fixed' and 'bug', or bug ID references in changelogs. Moreover, they would try to find the link by using features extracted from linked issues and commits. They obtained $89 \%$ precision and $78 \%$ recall on average. Nguyen et al. [20] presented MLink, a layered approach that exploits both textual and code-related features. They outperform ReLink by $13 \%$ to $17 \%$ on recall and $8 \%$ to $17 \%$ on precision [20]. However, they used only three projects when evaluating their work. Moreover, their results showed that some individual layer's precision or recall are very low. Finally, Schermann et al. [18] introduced PaLiMod to enable the analysis of interlinking characteristics in commit and issue data. They used this analysis to define their heuristics. PaLiMod achieves a precision of $96 \%$ and recall of $92 \%$ in the case of the Loner heuristic which are single commits with no link to the addressed issues. Also, their method reach overall precision of $73 \%$ with a recall of $53 \%$ in the case of the Phantom heuristic which are commits without a link in a series of commits that address a certain issue. Although the idea of the Phantom case was novel, the results were not significant compared to former heuristic methods such as MLink. One of the drawbacks of these studies is using a manually-created dataset by the authors themselves [21]. Most of these cases used manually labeled data which reduces the confidence in the results.

b) Machine-Learning-based approaches: The second approach to recovering links is to use traditional binary classifications, including RCLinker [3], FRLink [6] and PULink [1]. RcLinker employed ChangeScribe, a tool for creating a commit message and used a set of features to recover the links. They outperformed MLink in terms of F-measure by $138.66 \%$ [3]. ChangeScribe creates highly detailed commits which are not very suitable for feature extraction in this context. Recently, FRLink was introduced which uses its own set of features [6]. The authors also use complementary documents such as non-source documents to learn from relevant data They analyze and filter out irrelevant source code files to reduce data noise. FRLink outperforms RCLinker in Fmeasure by $40.75 \%$ when achieving the highest recalls. However, their approach encounter problems when (1) a dataset has a low percentage of non-source documents in commits, or (2) it has few or no similar code terms in the issue report and corresponding fixing commits. Also, text and code features were equally weighted in this approach. A close study to FRLink is PULink [1], where authors labeled their data as a True Link/unlabeled instead of True/False Links. They claim they can obtain the same value of precision and recall with almost $70 \%$ of the number of True Links in other approaches. However, they too had a problem when a dataset has a low percentage of True Links. Generally, the main problem of these studies is the low performance based on metrics like F1, precision, and recall. Although FRLink achieves higher recall scores, its precision and F1 are very low.

c) Deep-Learning-based approaches: Xie et al. [22] proposed DeepLink [22], which incorporates a knowledge based graph and deep learning to solve this problem. Using class embeddings in commit codes, the authors created this graph. Authors also use CBOW and Word2Vec embedding for commit and issue documentation. As we did not have access to the knowledge graph and replication package, we were not able to replicate this approach. Another publication also named DeepLink [2] uses a semantically-enhanced link recovery method based on deep neural networks to tackle this problem. The authors use recurrent neural networks on the textual information of issues and commits to train their model. They disregard comments because of their length and noise. They have added semantic to their model to have a better prediction. DeepLink outperforms FRLink in terms of F-measure by $10 \%$ [2]. The challenge with deep learning algorithms lies in the need for a large amount of data and high computational resources. Moreover, training these models takes a lot of time.

We propose a model that outperforms the baselines by exploiting information from both textual and non-textual channels. We use fewer resources and our training and prediction time are much lower. We also train with projects where fewer issues and commits are available. Thus our model will not fail when there is little historical data available for a project.

\section{CONCLUSION AND FutURE WORK}

The importance of recovering true connections between issues and their corresponding commits greatly affects various software maintenance tasks. Previous studies mostly focused on exploiting textual information to train their models to identify the links. However, we introduced a hybrid method, called Hybrid-Linker based on classical ML-based classifiers, that employs both textual and non-textual information to recover the links. For each project, we tune alpha, as an indication of the importance of each information channel. The results suggest that the non-textual information indeed help the predictions. This is highlighted in cases that there is little textual information available. Moreover, our approach requires shorter training time and outperforms both the competing methods, namely DeepLink [2] and FRLink [6] by $41.3 \%$ and $31.3 \%$ on F-measure, respectively.

In the future, we plan to boost our proposed classifier by identifying new features from different bug tracking and version control systems. We will also investigate other classifier architectures.

\section{ACKNOWLEDGEMENT}

We would like to acknowledge Mahtab Nejati for her valuable comments and help on this work. 


\section{REFERENCES}

[1] Y. Sun, C. Chen, Q. Wang, and B. Boehm, "Improving missing issuecommit link recovery using positive and unlabeled data," in 2017 32nd IEEE/ACM International Conference on Automated Software Engineering (ASE). IEEE, 2017, pp. 147-152.

[2] H. Ruan, B. Chen, X. Peng, and W. Zhao, "Deeplink: Recovering issuecommit links based on deep learning," Journal of Systems and Software, vol. 158, p. $110406,2019$.

[3] T.-D. B. Le, M. Linares-Vásquez, D. Lo, and D. Poshyvanyk, "Rclinker: Automated linking of issue reports and commits leveraging rich contextual information," in 2015 IEEE 23rd International Conference on Program Comprehension. IEEE, 2015, pp. 36-47.

[4] J. Anvik, L. Hiew, and G. C. Murphy, "Who should fix this bug?" in Proceedings of the 28th international conference on Software engineering, 2006, pp. 361-370.

[5] B. Dit, M. Revelle, M. Gethers, and D. Poshyvanyk, "Feature location in source code: a taxonomy and survey," Journal of software: Evolution and Process, vol. 25, no. 1, pp. 53-95, 2013.

[6] Y. Sun, Q. Wang, and Y. Yang, "Frlink: Improving the recovery of missing issue-commit links by revisiting file relevance," Information and Software Technology, vol. 84, pp. 33-47, 2017.

[7] M. Claes and M. V. Mäntylä, "20-mad: 20 years of issues and commits of mozilla and apache development," in Proceedings of the 17th International Conference on Mining Software Repositories, 2020, pp. 503-507.

[8] B. Pavlyshenko, "Using stacking approaches for machine learning models," in 2018 IEEE Second International Conference on Data Stream Mining \& Processing (DSMP). IEEE, 2018, pp. 255-258.

[9] J. H. Friedman, "Stochastic gradient boosting," Computational statistics \& data analysis, vol. 38, no. 4, pp. 367-378, 2002.

[10] S. Ting, W. Ip, A. H. Tsang et al., "Is naive bayes a good classifier for document classification," International Journal of Software Engineering and Its Applications, vol. 5, no. 3, pp. 37-46, 2011.

[11] L. Breiman, "Random forests," Machine learning, vol. 45, no. 1, pp. 5-32, 2001.

[12] T. Chen and C. Guestrin, "Xgboost: A scalable tree boosting system," in Proceedings of the 22nd acm sigkdd international conference on knowledge discovery and data mining, 2016, pp. 785-794.

[13] W. McKinney et al., "pandas: a foundational python library for data analysis and statistics," Python for High Performance and Scientific Computing, vol. 14, no. 9, pp. 1-9, 2011.

[14] S. Aiello, C. Click, H. Roark, L. Rehak, and P. Stetsenko, "Machine learning with python and h2o," H2O. ai Inc, 2016.

[15] F. Pedregosa, G. Varoquaux, A. Gramfort, V. Michel, B. Thirion, O. Grisel, M. Blondel, P. Prettenhofer, R. Weiss, V. Dubourg et al., "Scikit-learn: Machine learning in python," the Journal of machine Learning research, vol. 12, pp. 2825-2830, 2011.

[16] V. J. Hellendoorn and P. Devanbu, "Are deep neural networks the best choice for modeling source code?" in Proceedings of the 2017 11th Joint Meeting on Foundations of Software Engineering, 2017, pp. 763-773.

[17] A. Ampatzoglou, S. Bibi, P. Avgeriou, M. Verbeek, and A. Chatzigeorgiou, "Identifying, categorizing and mitigating threats to validity in software engineering secondary studies," Information and Software Technology, vol. 106, pp. 201-230, 2019.

[18] G. Schermann, M. Brandtner, S. Panichella, P. Leitner, and H. Gall, "Discovering loners and phantoms in commit and issue data," in 2015 IEEE 23rd International Conference on Program Comprehension. IEEE, 2015, pp. 4-14.

[19] R. Wu, H. Zhang, S. Kim, and S.-C. Cheung, "Relink: recovering links between bugs and changes," in Proceedings of the 19th ACM SIGSOFT symposium and the 13th European conference on Foundations of software engineering, 2011, pp. 15-25.

[20] A. T. Nguyen, T. T. Nguyen, H. A. Nguyen, and T. N. Nguyen, "Multilayered approach for recovering links between bug reports and fixes," in Proceedings of the ACM SIGSOFT 20th International Symposium on the Foundations of Software Engineering, 2012, pp. 1-11.

[21] T. F. Bissyande, F. Thung, S. Wang, D. Lo, L. Jiang, and L. Reveillere, "Empirical evaluation of bug linking," in 2013 17th European Conference on Software Maintenance and Reengineering. IEEE, 2013, pp. 89-98.

[22] R. Xie, L. Chen, W. Ye, Z. Li, T. Hu, D. Du, and S. Zhang, "Deeplink: A code knowledge graph based deep learning approach for issue-commit link recovery," in 2019 IEEE 26th International Conference on Software
Analysis, Evolution and Reengineering (SANER). IEEE, 2019, pp. 434 444. 